\section{Peritonitis associated with vaginal leakage of dialysis fluid in continuous ambulatory peritoneal dialysis}

As the use of continuous ambulatory peritoneal dialysis for the treatment of end-stage renal failure increases so rarer complications of this treatment are being recognised. ${ }^{1}$ We report a case of peritonitis with leakage of dialysis fluid from the peritoneum by way of the Fallopian tubes and uterus into the vagina.

\section{Case report}

This 17-year-old girl had been born with an imperforate anus and rectovaginal fistula, which was treated by a rectal cut-back. Because of recurrent urinary tract infections associated with ureteric reflux and hydronephrosis bilateral ureteric reimplantation and an ileal loop bladder were performed in childhood. She was treated with haemodialysis at 15 years of age because of reduced renal function. A year later she received a cadaver transplant which drained into the ileal loop. At this operation interperitoneal adhesions were present but the Fallopian tubes were normal. She was again treated with haemodialysis six weeks later after a transplant nephrectomy for severe irreversible rejection. Because of problems of vascular access continuous ambulatory peritoneal dialysis was begun in October 1980.

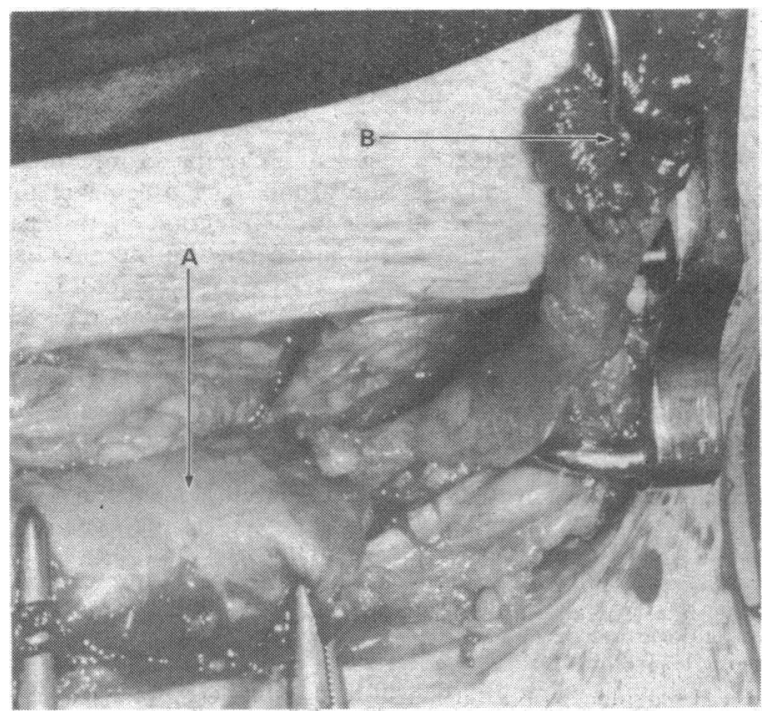

Laparotomy photograph showing uterus (A) and enlarged lef Fallopian tube with a probe in the dilated lumen (B).

She had had her menarche aged 13 but had secondary amenorrhoea from the age of $15 \frac{1}{2}$ until she menstruated again in December 1980 two and a half months after starting continuous ambulatory peritoneal dialysis. Three days after menstruation she developed a coagulase-negative staphylococcal peritonitis which was treated with gentamicin and cloxacillin. During the next three months she had recurrent infections due to coagulase-negative staphylococci with different antibiotic sensitivities. In March 1981 Streptococcus faecalis was isolated from the peritoneal fluid and the infection was treated successfully with ampicillin. In April 1981 she complained of leakage of peritoneal fluid from the vagina, which was confirmed by intraperitoneal injection of methylene blue. Further infection with coagulasenegative staphylococci and $S$ faecalis failed to respond to antibiotics. Vaginal leakage continued and peritonitis due to Candida albicans developed in July 1981. At laparotomy the peritoneal cavity was reduced with numerous adhesions and the peritoneum was grossly thickened. The left Fallopian tube was enlarged with a dilated lumen and appeared to be the site of the fistula (see figure). Sterilisation was performed by ligation, the medial ends of the tubes being buried in the broad ligament. She was subsequently treated with haemodialysis using a subclavian cannula. On restarting peritoneal dialysis four weeks later there was no further vaginal leakage or peritonitis. Nevertheless, the peritoneal cavity was decreased considerably in size, thus giving inadequate dialysis. Haemodialysis was, therefore, restarted using a Gortex graft in October 1981.

\section{Comment}

Peritonitis is still a major cause of morbidity and mortality with continuous ambulatory peritoneal dialysis. ${ }^{1}$ Coagulase-negative staphylococci account for about $40 \%$ of cases; they probably result from accidental contamination and usually have a good response to antibiotic treatment. ${ }^{2}$

In a report of a large series of patients undergoing continuous ambulatory peritoneal dialysis ${ }^{1}$ a similar instance of vaginal leakage occurred in a patient who also had candidal peritonitis. This was treated by temporarily removing the dialysis cannula and ligating the Fallopian tubes.

Our patient had recurrent peritonitis despite a good technique of bag-exchange. The cause of the initial episodes of peritonitis before the appearance of the vaginal leakage is uncertain, but these attacks were precipitated by menstruation and perhaps retrograde passage of infected material. Retrograde flow of infection with peritoneal spread is well known in cases of salpingitis. ${ }^{3}$ In patients undergoing continuous ambulatory peritoneal dialysis the dialysis fluid may become blood-tinged during menstruation, which can result in eosinophilic peritonitis, ${ }^{4}$ which our patient did not have.

Vaginal leakage of uninfected dialysis fluid through a structurally normal genital tract is unknown. The cause of the Fallopian tube damage and dilatation is uncertain but may have been related to the peritonitis. We recommend that in patients who develop a vaginal leak of dialysis fluid a cause should be looked for and corrected immediately, as delay may lead to loss of peritoneal surface from prolonged peritonitis.

${ }^{1}$ Khanna R, Oreopoulos DG, Dombros N, et al. CAPD after three years. till a promising treatment. Peritoneal Dialysis Bulletin 1981;1:24-34. 2 Vas S. Peritonitis during CAPD. Peritoneal Dialysis Bulletin 1981;1:47-9.

3 Jeffcoate N. Principles of gynaecology. 4th ed. London: Butterworth, 1975:320.

4 Gokal R, Ramos JM, Ward MK, Kerr DNS. Eosinophilic peritonitis in continuous ambulatory peritoneal dialysis (CAPD). Clin Nephrol 1981 ; $15: 328-30$.

(Accepted 5 February 1982)

Departments of Renal Medicine and Surgery, Manchester Royal Infirmary, Manchester M13 9WL

R A COWARD, BMEDSCI, MRCP, research fellow

R GOKAL, MD, MRCP, consultant physician

$M$ WISE, FRCS, tutor in surgery

N P MALLICK, FRCP, consultant physician

St Mary's Hospital, Manchester M13 9WL

D WARRELL, MD, FRCOG, consultant obstetrician and gynaecologist

\section{Recurrent trimethoprim-associated fixed skin eruption}

I describe a case of fixed skin eruption associated with ingestion of trimethoprim in a patient known to be allergic to co-trimoxazole. This case emphasises that sulphamethoxazole should not always be assumed to be the culprit in co-trimoxazole-associated cutaneous reactions. I am not aware of any previous reports of a fixed skin eruption associated with trimethoprim used as a single agent.

\section{Case report}

A 49-year-old woman presented with a history of recurrent attacks of a well-circumscribed, non-irritating area of swelling at a fixed site on the left side of her neck. She had suffered eight or nine self-limiting attacks in the past two years and could definitely associate one of the episodes with ingestion of co-trimoxazole (trimethoprim plus suphamethoxazole). She had been taking propranolol $40 \mathrm{mg} /$ day, cyclopenthiazide $0.25 \mathrm{mg} /$ day, and glyceryl trinitrate for 18 months for hypertension and angina, but there was no apparent direct association between these drugs and her skin problem. She also frequently took antibiotics for recurrent urinary tract infections.

Initial examination showed evidence of her previous attacks-namely, 
a well-circumscribed macular area of post-inflammatory pigmentation 3-4 cm in diameter-on the left side of her neck.

Although given a warning about the nature of the problem and an explanation that co-trimoxazole had probably been associated with at least one of her previous attacks, she was reviewed in the clinic two months later with a recurrence of the lesion, which had flared within one day of her taking co-trimoxazole for a urinary infection. Six months later she was seen again with a recurrence of her lesion, which had developed within one day of her taking trimethoprim as a single agent for yet another urinary infection. There did not seem to be any association between the occurrence of urinary infections and the appearance of her skin eruption.

\section{Comment}

Trimethoprim as a single agent has been available in the United Kingdom since 1979, its main indication being the treatment of urinary tract infections. Lacey et al suggested that it may have fewer adverse effects, including cutaneous reactions, associated with its use than co-trimoxazole ${ }^{1}$ owing to the absence of the sulphamethoxazole component. Although this may be true, cutaneous reactions do occur with trimethoprim as shown by this patient. Furthermore, I have seen three other patients in the past year in whom an erythematous maculopapular eruption occurred after treatment with trimethoprim.

The manufacturers of trimethoprim and the Committee on Safety of Medicines have had no reports of trimethoprim-associated fixed drug eruptions.

${ }^{1}$ Lacey RW, Lord VL, Gunasekera HKW, Leiberman PJ, Luxton DEA. Comparison of trimethoprim alone with trimethoprim sulphamethoxazole in the treatment of respiratory and urinary infections with particular reference to selection of trimethoprim resistance. Lancet 1980 ;i 1270-3.

(Accepted 24 February 1982)

Skin Department, The London Hospital, London E1 1BB

JOHN R GIBSON, MB, MRCP, honorary senior registrar

\section{Meningitis and recurrent septicaemia secondary to unsuspected pacemaker infection}

Septicaemia is a well-known, though uncommon, complication of cardiac pacemaker implantation. It usually follows local infection of the generator or wire with bacteria from the skin. Staphylococcus aureus is the most common bacterium isolated. ${ }^{12}$ We report a case of meningitis and recurrent septicaemia due to Klebsiella aerogenes in which the pacemaker was not initially suspected as the source of infection.

\section{Case report}

On 27 February 1980 a 73 -year-old man with diabetes mellitus had a pacemaker inserted for complete heart block. A five-day course of prophylactic flucloxacillin and amoxycillin was started. That evening he became feverish and was later found to have a urinary tract infection with an amoxycillin-resistant klebsiella, which was subsequently treated with co-trimoxazole.

On 27 August he was admitted to the Royal United Hospital, Bath, after two weeks of intermittent fever and rigors. On the day of admission his temperature rose to $39^{\circ} \mathrm{C}$, but there were no localising signs, particularly none at the pacemaker site. Blood cultures yielded $\mathrm{Kl}$ aerogenes sensitive to gentamicin and cefuroxime (see table). Urine cultures were sterile. Treatment was started with ampicillin and gentamicin but was later changed to cefuroxime $750 \mathrm{mg}$ three times daily intramuscularly, and his temperature returned to normal.

On 3 September, despite continuing cefuroxime, his fever returned and he developed clinical meningitis with neck stiffness, Kernig's sign, and a depressed level of consciousness. The cerebrospinal fluid contained $700 \times 10^{6}$ leucocytes $/ 1\left(700 / \mathrm{mm}^{3}\right)\left(80^{\circ} \%\right.$ neutrophils $), 100 \times 10^{6}$ red cells $/ 1$ $\left(100 / \mathrm{mm}^{3}\right)$, and $1.6 \mathrm{~g}$ protein $/ 1$. Though a blood culture yielded $K l$ aerogenes, no micro-organisms were seen in a Gram-stained film of the cerebrospinal fluid and there was no growth after 36 hours' culture. Estimation of cerebrospinal fluid glucose concentration was unhelpful as he was taking insulin. He was given intravenous gentamicin and co-trimoxazole $10 \mathrm{ml}$ (trimethoprim $160 \mathrm{mg}$ and sulphamethoxazole $800 \mathrm{mg}$ ) twice a day and the cefuroxime was stopped. Three days later, though the cerebrospinal fluid leucocyte count had dropped to $35 \times 10^{6} / 1\left(35 / \mathrm{mm}^{3}\right)$, his clinical condition remained poor, and treatment was changed to intravenous gentamicin and cefuroxime $1.5 \mathrm{~g}$ three times daily with intrathecal gentamicin $5 \mathrm{mg}$ daily for eight days. His condition improved and the cerebrospinal fluid leucocyte count returned to normal. All antibiotics were stopped on 25 September. On 29 September septicaemia recurred and $\mathrm{Kl}$ aerogenes with reduced sensitivity to gentamicin was isolated from blood cultures. Gentamicin and cefuroxime were restarted.

An intensive search for the source of infection had meanwhile been made. Intravenous urography, barium enema, cholecystography, abdominal ultrasonography, and liver scanning were all unhelpful. A gallium-67 scan, however, showed an increase in uptake below the pulse generator. He was therefore transferred to the Bristol Royal Infirmary, where the pulse generator and, later, all but the proximal tip of the pacemaker wire were removed. The wire had fractured in the right atrium and the tip remained embedded in the right ventricle. More than one strain of $K l$ aerogenes was isolated from the pacemaker wire and from blood cultures (see table). His temperature returned to normal, and antibiotics were stopped on 23 October.

On 30 October his fever returned and highly resistant $K l$ aerogenes was isolated from blond cultures. He was treated with cefotaxime $1 \mathrm{~g}$ three times daily intravenously, and the remaining wire was removed in early November After this the fever settled, and cefotaxime was stopped 10 days after operation. A new endocardial pacing system was inserted and he made a full recovery.

\section{Comment}

When septicaemia occurs as a result of a pacemaker infection there is usually evidence of local infection around the pacemaker unit. A gallium scan was required to detect local infection in this patient. We assume that $\mathrm{Kl}$ aerogenes were initially carried in the blood stream from the urinary tract.

Antibiotics failed to erradicate the infection and merely selected more resistant strains of $K l$ aerogenes, which subsequently caused clinical septicaemia. This case emphasises the need to remove the entire pacing system when an infection like this occurs and thereby supports recommendations made by other workers. ${ }^{12}$

We thank Dr J Russell Rees and Dr Diana White for help in preparing this report and Dr Mortimer, Coventry Public Health Laboratory, fo serotyping the klebsiella isolates and confirming their identity.

${ }^{1}$ Morgan G, Ginks W, Siddons H, Leatham A. Septicemia in patients with an endocardial pacemaker. Am $\mathcal{F}$ Cardiol $1979 ; 44: 221-4$

${ }^{2}$ Choo MH, Holmes DR, Gersh BJ, et al. Permanent pacemaker infections : characterization and management. Am $\mathcal{F}$ Cardiol 1981;48:559-64.

(Accepted 24 February 1982)

Royal United Hospital, Bath BA1 3NG

E SOUTHALL, MB, MRCP, medical registrar

R D THOMAS, MD, MRCP, consultant physician

Bristol Royal Infirmary, Bristol BS2 8HW

P R WILKINSON, MB, MRCP, senior medical registrar

E W WILLIAMS, MB, MRCPATH, consultant senior lecturer in bacteriology

Minimum inhibitory concentrations (agar incorporation method) and capsular serotypes of isolates of Klebsiella aerogenes

\begin{tabular}{|c|c|c|c|c|c|c|c|}
\hline \multirow{2}{*}{ Isolate } & \multicolumn{6}{|c|}{ Minimum inhibitory concentrations of antibiotics $(\mathrm{mg} / \mathrm{l})$} & \multirow{2}{*}{$\begin{array}{l}\text { Capsular } \\
\text { serotypes }\end{array}$} \\
\hline & Gentamicin & Cefuroxime & Cefotaxime & $\begin{array}{l}\text { Sulpha- } \\
\text { methoxazole }\end{array}$ & Trimethoprim & $\begin{array}{c}\text { Trimethoprim/ } \\
\text { sulphamethoxazole }\end{array}$ & \\
\hline $\begin{array}{l}1 \text { (Blood culture, } 27 \text { Aug 1980) } \\
2 \text { (Blood culture, } 29 \text { Sept 1980) } \\
3 \text { (Blood culture, } 13 \text { Oct 1980) } \\
4 \text { (Blood culture, 13 Oct 1980) } \\
5 \text { (Pacemaker wire, } 17 \text { Oct 1980) } \\
6 \text { (Pacemaker wire, 17 Oct 1980) } \\
7 \text { (Blood culture, } 30 \text { Oct 1980) }\end{array}$ & $\begin{array}{l}0 \cdot 5 \\
2 \\
8 \\
1 \\
2 \\
2 \\
2\end{array}$ & $\begin{array}{r}2 \\
1 \\
8 \\
8 \\
1 \\
16 \\
128\end{array}$ & $\begin{array}{l}0 \cdot 03 \\
0 \cdot 03 \\
0 \cdot 06 \\
0 \cdot 03 \\
0 \cdot 03 \\
0 \cdot 25 \\
1\end{array}$ & $\begin{array}{l}16 \\
16 \\
16 \\
16 \\
16 \\
16 \\
32\end{array}$ & $\begin{array}{l}0 \cdot 25 \\
0 \cdot 25 \\
0 \cdot 5 \\
0 \cdot 5 \\
0 \cdot 25 \\
2 \\
16\end{array}$ & $\begin{array}{l}0 \cdot 12: 2 \cdot 5 \\
0 \cdot 12 / 2 \cdot 5 \\
0 \cdot 12: 2 \cdot 5 \\
0 \cdot 12 \cdot 2 \cdot 5 \\
0 \cdot 12 \cdot 2 \cdot 5 \\
0 \cdot 12 \cdot 2 \cdot 5 \\
\quad 1: 20\end{array}$ & $\begin{array}{l}\text { Type 1 } \\
\text { Type 1 } \\
\text { Type 1 } \\
\text { Not typable } \\
\text { Not typable } \\
\text { Type 1 } \\
\text { Not typable }\end{array}$ \\
\hline
\end{tabular}

